\title{
FBG Sensor Probes with Silver Epoxy for Tracing the Maximum Strain of Structures
}

\author{
Jooeun Im*, Mihyun Kim*, Ki-Sun Choi**, Tae-Kyung Hwang*** and Il-Bum Kwon* ${ }^{\dagger}$
}

\begin{abstract}
Structures can be evaluated their health status by allowable loading criteria. These criteria can be determined by the maximum strain. Therefore, in order to detect this maximum strain of structures, fiber optic Bragg grating(FBG) sensor probes are newly designed and fabricated to perform the memorizing detection even if the sensor system is on-and-off. The probe is constructed with an FBG optical fiber embedded in silver epoxy. When the load is applied and removed on the structure, the residual strain remains in the silver epoxy to memorize the maximum strain effect. In this study, a commercial Al-foil bonded FBG sensor probe was tested to investigate the detection feasibility at first. FBG sensor probes with silver epoxy were fabricated as three different sizes. The detection feasibility of maximum strain was studied by doing the tensile tests of CFRP specimens bonded with these FBG sensor probes. It was investigated the sensitivity coefficient defined as the maximum strain divided by the residual strain. The highest sensitivity was 0.078 of the thin probe having the thickness of $2 \mathrm{~mm}$.
\end{abstract}

Keywords: Sensor Probe, FBG Sensor, Composite, Residual Strain

\section{Introduction}

Structures can be evaluated their health status with allowable loading criteria. These criteria can be determined by the maximum strain. Therefore, in order to detect this maximum strain of structures, FBG sensor probes are newly designed and fabricated to perform the detection without any measurement uncertainty even if the sensor system is on-and-off. The maximum strain of structural materials can be traced by measuring the residual strain in the probe. FBG sensors have been developed for applying the field of structural health monitoring, such as, strain measurements, failure diagnostics, thermal measurements, pressure monitoring. FBGs have many advantages, for example, electro-magnetic resistance, small size, resistance to corrosion, multiplexing of sensors along a single fiber. Moreover, FBGs can be used for the measure- ment of parameters manifesting as the changes of strain or temperature [1-4].

Carbon fiber reinforced plastics (CFRP) are used for almost modern commercial aircrafts as a primary structural component. For structural health monitoring, the surface mounted or embedded FBG sensors have been replaced strain gauges [7]. In order to monitor the health status of composite materials, the maximum strain detection is very important because we can determine the structural safety criteria from this information. However, CFRP is fully recovered their strain after removing the loading. Therefore, the maximum strain of CFRP is not easy to detect without continuous measurement. However, if an FBG probe is coated or bonded with some plastic materials, this probe will have some amount of the residual strain after experiencing maximum strain. Therefore, if we know the relation between residual strain and maximum strain, the maximum strain can be

[Received: September 10, 2013, Revised: October 22, 2013, Accepted: October 23, 2013] *Korea Research Institute of Standards and Science, Daejeon 305-340, Korea, **FINISA Korea Co. Ltd., Gwangju 500-460, Korea, ***Agency for Defence Development, Daejeon 305-152, Korea, †Corresponding Author: ibkwon@kriss.re.kr (c) 2013, Korean Society for Nondestructive Testing 
determined after sensing the residual strain of the probe.

There are many coating optical fibers to protect bare optical fibers because these bare optical fibers are usually fragile. To protect the glass optical fibers from moisture attack, metallic coatings are applied on optical fibers, such as, aluminum, indium, tin, antimony, zinc, lead, copper, nickel, and gold [5]. Some of these fibers exhibit higher resistance to moisture attack and significantly higher strength than polymercoated fibers, and some of these fibers can withstand relatively higher temperature.

On the one hand, optical fibers are coated to improve mechanical properties, such as, Young's modulus, thermal expansion coefficient and Poisson's ratio, enhance reliability and protect against harsh environment applications [6]. Although these coating techniques of optical fibers have been improved the properties of optical fibers, there were no studies on the maximum strain detection of structures using the residual strain of coating materials.

Therefore, we designed and fabricated new FBG sensor probes embedded in silver epoxy molding that can trace the maximum strain of structural materials by the residual strain. The feasibility was studied using Al-foil bonded FBG probes. The detection sensitivity was investigated by doing tensile tests of CFRP specimens with the FBG probes with silver epoxy.

\section{Detection Principle of an FBG Probe with Silver Epoxy}

A new sensing probe based on the principle of FBG sensors is proposed for detecting the maximum tensile strain of structures. This probe is designed with an FBG fiber embedded in silver epoxy shown in Fig. 1. This figure also shows how the probe is operated when the maximum strain is induced on the structure.

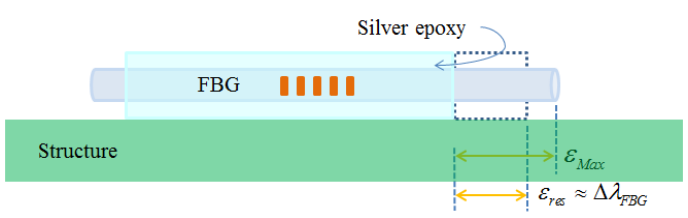

Fig. 1 Schematics of an FBG probe with silver epoxy

This probe should be attached on the surface of the structure to detect the maximum strain also shown in Fig. 1. When the load is applied on the host structural material, the silver epoxy and the FBG fiber are extended to the maximum strain, $\varepsilon_{\text {MAX }}$. Also, when the load is removed, then the silver epoxy with an FBG fiber will have some residual strain, $\varepsilon_{r e s}$, according to the force equilibrium between the host structural material and the silver epoxy. Therefore, this residual strain can be detected by the FBG fiber in the silver epoxy shown in Fig. 1. In order to use this sensing technique, the sensitivity coefficient, the ratio between the relation between the residual and maximum strain, is defined as:

$$
C_{\text {sen }}=\frac{\varepsilon_{\text {res }}}{\varepsilon_{\max }}
$$

Where $\varepsilon_{M A X}$ is the maximum strain, $\varepsilon_{\text {res }}$ is the residual strain of the sensor probe. If the residual strain is proportionally remained with the maximum strain in the probe, then the maximum strain can be determined by using Eq. 1 .

The physical dimension of the probe can influence the sensitivity coefficient because the residual strain is determined by the force equilibrium between the probe and the host structural material.

\section{Feasibility Study Using an Al-Foil Bonded FBG Probe}

The probe should be able to sense the residual strain in order to detect the structural maximum strain. Therefore, the sensitivity 


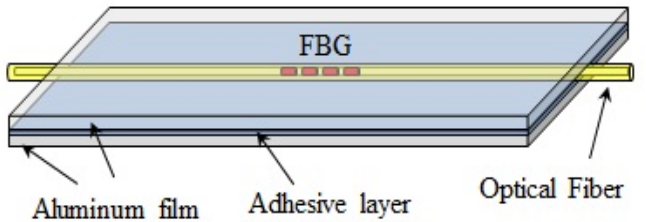

Fig. 2 An aluminum bonded FBG probe

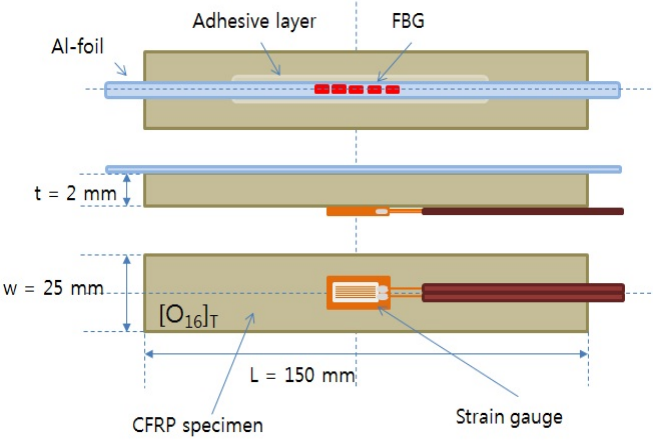

Fig. 3 Schematic diagram of an Al-foil bonded FBG sensor probe on a CFRP specimen.

coefficient should be studied for the probe with plastic materials. An Al-foil bonded FBG sensor probe is designed as one film shape of an FBG inserting between two Al-foils, the thickness of each foil is $36 \mu \mathrm{m}$, shown in Fig. 2. There is an FBG sensor probe, which is fully bonded on the surface of structures shown in the upper part of Fig. 2. Also, the probe is fabricated with commercially available aluminum foil of the thickness, $18 \mu \mathrm{m}$, bonded on an FBG using instant adhesive (CC-33A, cyano-acrylate base, Kyowa). To study the feasibility of these probes, CFRP specimen, $\left[0_{16}\right]_{\mathrm{T}}$, was prepared as $2 \mathrm{~mm}$ thickness, $25 \mathrm{~mm}$ width and $150 \mathrm{~mm}$ length shown in Fig. 3. Al-foil bonded FBG sensor probe was bonded on the upper surface, and the $5 \mathrm{~mm}$ length of electric strain gauge was also bonded on the bottom surface of the specimen.

Fig. 4 shows the specimen with a fully bonded FBG sensor. Universal testing machine (UTM) was used to induce the strain on the specimens, this machine was controlled as the condition of constant displacement speed, $1 \mathrm{~mm} / \mathrm{min}$, and the

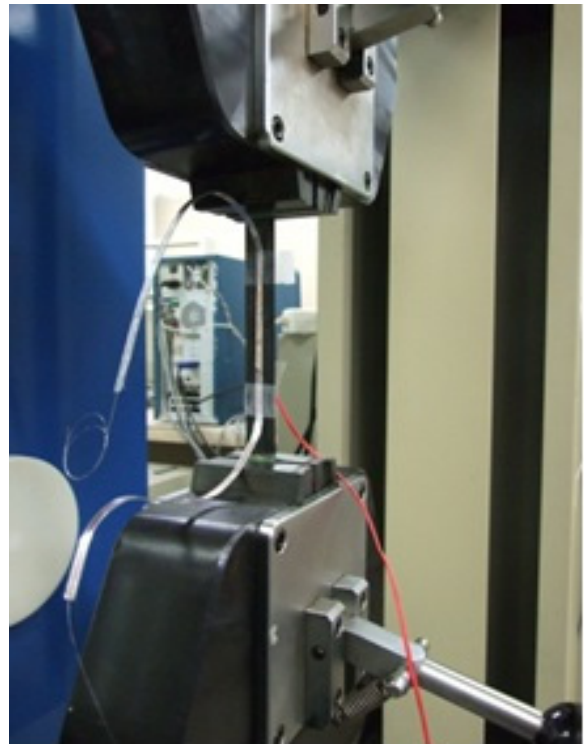

Fig. 4 A CFRP specimen with an Al-foil bonded FBG sensor in universal testing machine

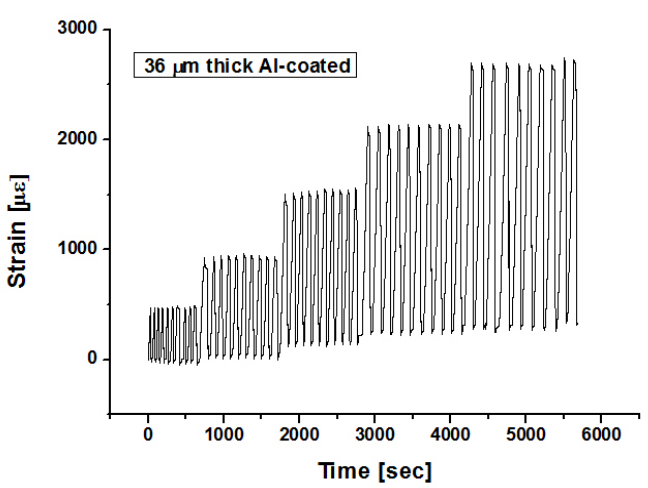

(a) Strain history

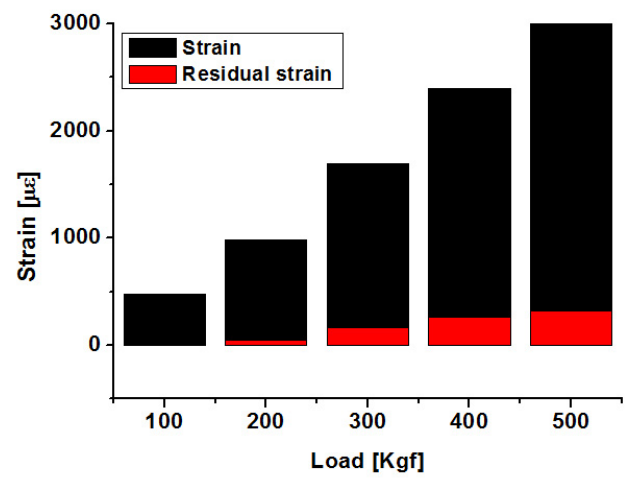

(b) Maximum and residual strain

Fig. 5 Strain history and maximum/residual strain of the Al-foil bonded FBG probe 
load was increased as the step of $100 \mathrm{kgf}$. Each load step was loaded and unloaded about 10 times. The load was unloaded until zero value to confirm the residual strain at each step. The overall setup is composed of UTM and its controlled computer, an FBG interrogator and specimen. The FBG sensor data is acquired by the FBG interrogator (CyTroniq) and the electric strain gauge data is also gathered through an $\mathrm{A} / \mathrm{D}$ converter from the strain gage conditioner.

The maximum and residual strains are acquired from this Al-bonded FBG probe and shown in Fig. 5. The strain was remained at second loading step. It means that the residual strain can be generated at lower strain of 1000 micro-strain. When the maximum strain is increased, the residual strain is also increased. The sensitivity coefficient between the maximum and residual strain is calculated as 0.15 .

\section{Detection Sensitivity of FBG Probes Embedded in Silver Epoxy}

The FBG probe with silver epoxy is designed referring from the reference [8] as shown in Table 1. These probes are designed to investigate the effect of FBG location and the width of the probes on the sensitivity coefficient as shown in Fig. 6.

Silver epoxy is prepared to be fabricated with an FBG optical fiber because this silver epoxy of about $10 \mathrm{GPa}$ elastic modulus, can be plastically deformed when the host material of about $72 \mathrm{GPa}$ elastic modulus experiences the maximum strain. This silver epoxy is a popularly commercialized product as a conductive and thermal polymer and it can be cured in room-temperature. The probes are fabricated as the following procedural steps. At first, silver epoxy is poured into a silicon mold. Secondly, an FBG fiber is positioned at the center or the bottom of the mold to the thickness direction. At third step, These are cured in the oven at
Table 1 Physical dimension of FBG probes
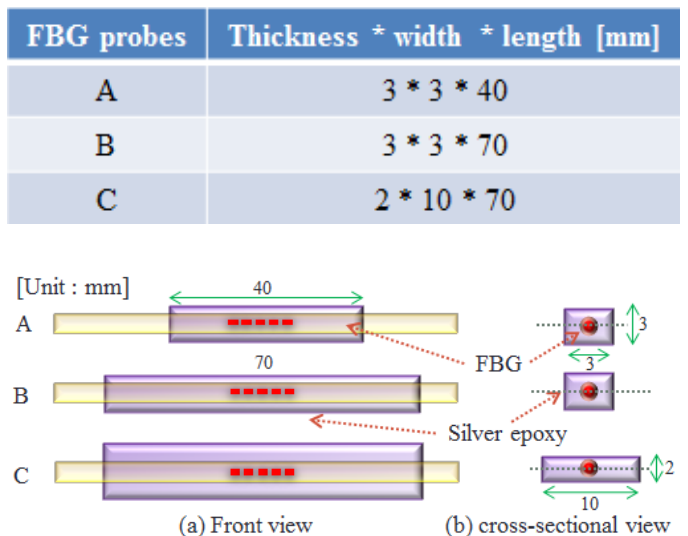

Fig. 6 Schematics of FBG probes with silver epoxy

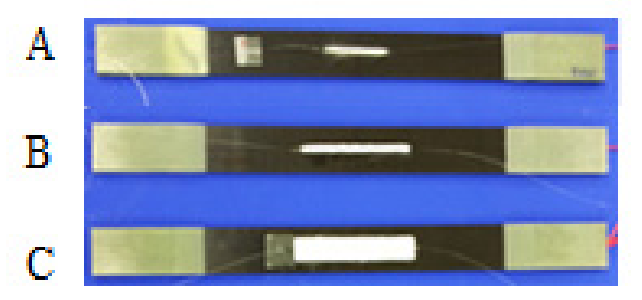

Fig. 7 FBG probes with silver epoxy on CFRP specimens

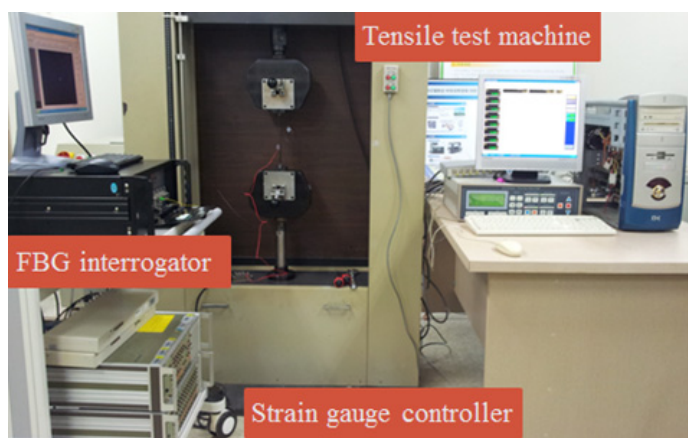

Fig. 8 Experimental setup for tensile test

$100^{\circ} \mathrm{C}$ during almost 1 hour for fast hardening. These probes are finally attached on the surfaces of each CFRP specimen for tensile test.

The fabricated FBG probes are attached on the surfaces of CFRP specimens shown in Fig. 7. The thickness, width and length of probe A is $3 \mathrm{~mm}, 3 \mathrm{~mm}, 40 \mathrm{~mm}$ and the FBG position is at the center of the probe. The size of probe 


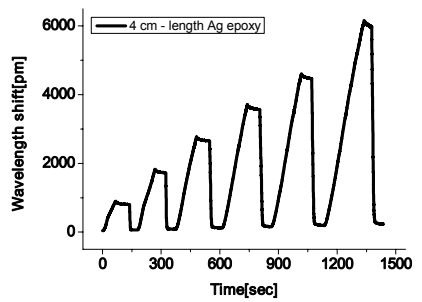

(a) Probe A

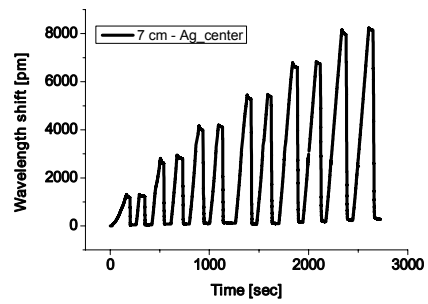

(b) Probe $B$

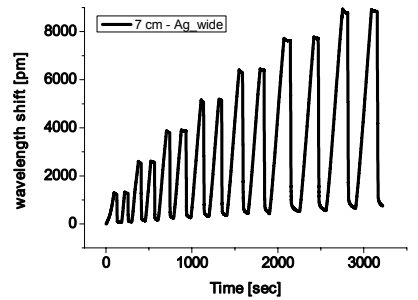

(c) Probe $\mathrm{C}$

Fig. 9 Strain history of FBG probes with silver epoxy

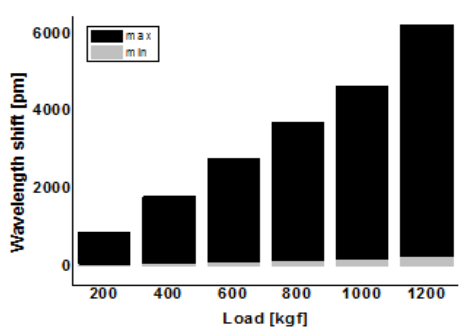

(a) Probe A

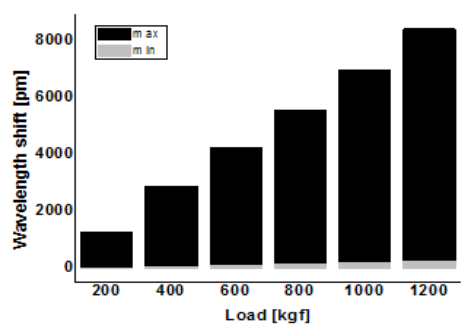

(b) Probe B

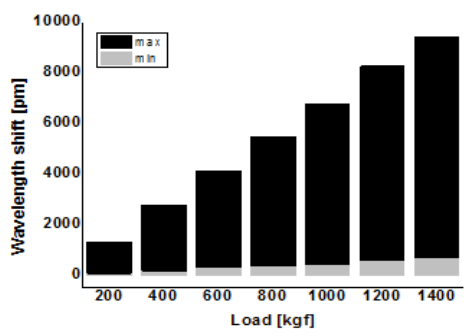

(c) Probe C

Fig. 10 Residual strain change of each load step

B is $3 \mathrm{~mm} \mathrm{-} \mathrm{thick,} 3 \mathrm{~mm}$ - wide, $70 \mathrm{~mm} \mathrm{-}$ long and the FBG position is at the bottom of the probe. The size of probe $\mathrm{C}$ is $2 \mathrm{~mm}$ thick, $10 \mathrm{~mm}$ - wide, $70 \mathrm{~mm} \mathrm{-} \mathrm{long} \mathrm{and} \mathrm{the}$ FBG position is at the center of the probe.

Tensile tests are performed by UTM and its control computer. An FBG interrogator and an electrical strain gage signal controller etc are also used as shown in Fig. 8. FBG sensor data are acquired by the FBG interrogator (CyTroniq) and electric strain gauge data are also acquired by the signal controller through A/D converter. The load step is $100 \mathrm{kgf}$ and the displacement control speed is $1 \mathrm{~mm} / \mathrm{min}$ and the data acquisition speed is $10 \mathrm{~Hz}$. The electrical strain gauge and FBG sensor data are acquired simultaneously. The load is increased and removed repeatedly to confirm how the residual strain is remained.

The strain history is shown in Fig. 9 according to the loading and unloading process. The red dashed line shows the slope of the residual strain due to time, the increase of the loading. In this figure, the probe $\mathrm{A}$ and $\mathrm{C}$ have similar residual strain revels, the highest residual strain is shown in the probe D. At each load step, the residual strain is well presented as the bar graph shown in Fig. 10. The sensitivity coefficients of each probe are $0.037,0.032$ and 0.078 , respectively.

In this study, it was studied that the maximum strain of host structural materials can be detected by the proposed FBG sensor probe with silver epoxy in the condition of static loading condition. If this technique is studied more precisely at the loading history and the loading speed on the sensitivity coefficient of the probes, then this technique is also applied on the dynamic loading condition.

\section{Conclusion}

A new FBG sensor probe is developed in order to detect the maximum strain induced in structures at any time, even when the sensor system is on and off status periodically. This probe uses the plasticity of materials with an 
FBG optical fiber in order to memorize the maximum strain effect. The plastic material should be plastically deformed by the maximum strain of the host material. In order to detect the maximum strain, this plastic material should be able to remain some amount of their residual strain even if the maximum strain of the host material is fully released. On the feasibility study using Al-bonded FBG probe, it is confirmed that the residual strain of the probe can indicate the maximum strain of CFRP structural materials. FBG sensor probe with silver epoxy is designed and fabricated to detect the maximum strain of CFRP composites. The sensitivity coefficient is an important parameter to determine the maximum strain by measuring the residual strain. The $10 \mathrm{~mm}$ width probe is mostly sensitive and the sensitivity coefficient is 0.078. The measurement range, resolution, and repeatability should be investigated to apply this technique on various industrial fields.

\section{Acknowledgment}

This work was supported by a project (ADD-11-01-05-11) of Agency for Defense Development.

\section{References}

[1] S. C. Her and C. Y. Huang, "Effect of coating on the strain transfer of optical fiber sensors," Sensors, Vol. 11, No. 7, pp. 6926-6941 (2011)

[2] F. Ansari and L. Yuan, "Mechanics of bond and interface shear transfer in optical fiber sensors," Journal of Engineering Mechanics, Vol. 124, No. 1, pp. 358-394 (1998)

[3] Q. Li, G. Li and G. Wang, "Effect of the plastic coating on strain measurement of concrete by fiber optic sensor," Measurement, Vol. 34, No. 3, pp. 215-227 (2003)

[4] A. Othonos, "Fiber Bragg gratings," Rev. Sci. Instrum., Vol. 68, No. 12, pp. 43094341 (1997)

[5] R. R. Kumar, M. Suesser, K. G. Narayankhedrar, G. Krieg and M. D. Atrey, "Performances evaluation of metalcoated fiber Bragg grating sensors for sensing cryogenic temperature," Cryogenics, Vol. 48, pp. 142-148 (2008)

[6] R. Sekar, B. N. Shivanaju, K. P. Lakshmi and S. Asokan, "Dual functional performance of fiber Bragg gratings coated with metals using flash evaporation technique," Optical Fiber Technology, Vol. 18, pp. 183-185 (2012)

[7] F. Surre, R. H. Scott, R. Banerji, P. A. M. Basheer, T. Sun and K. T. V. Grattan, "Study of reliability of fiber Bragg grating fibre optic strain sensors for field-test applications," Sensors and Actuators A, Vol. 185, pp. 8-16 (2012)

[8] I.-B. Kwon, K.-S. Choi, G.-J. Kim, D.-J. Kim, Y.-H. Huh and D.-J. Yoon, "Feasibility study on packaged FBG sensors for de-bonding monitoring of composite wind turbine blade," Journal of the Korean Society for Nondestructive Testing, Vol. 31, No. 4, pp. 382-390 (2011) 